\title{
A Two-Level Temporal Fair Scheduler for Multi-Cell Wireless Networks
}

\author{
Shahram Shahsavari and Nail Akar
}

\begin{abstract}
We propose a two-level scheduler for a frequency reuse-1 multi-cell wireless network satisfying inter- and intra-cell weighted temporal fairness constraints. As opposed to hard partitioning of the entire frequency band to different cell patterns in frequency reuse- $M$ systems $(M>1)$, we propose sharing this band opportunistically in time by these patterns. Through numerical examples, we show notable gains in overall network throughput due to improved multi-user diversity in comparison with a conventional frequency reuse- 3 system.
\end{abstract}

Index Terms-Multi-cell scheduling, temporal fairness.

\section{INTRODUCTION}

$\mathbf{T}$ RADITIONAL cellular wireless networks employ frequency reuse with parameter $M>1$ in which the frequency band is partitioned into $M$ subbands. Each of these subbands is then allocated to individual cells comprising a Base Station (BS) and multiple users [1]. The reuse parameter $M$ determines the distance between any two closest interfering cells using the same subband. Typical values of $M$ are 3, 4, or 7 [1]. The set of cells with the same subband assignment is called a (transmission) pattern due to the way these cells form a regular pattern in the 2D cell-layout. Note that cells in the same pattern can transmit simultaneously in the same subband. In frequency reuse- $M$ networks with $M>1$, users' scheduling decisions are made locally for each cell by the BS without a need for coordination among cells, thus referred to as single-cell scheduling. Within a single cell, opportunistic scheduling is employed that exploits the time-varying characteristics of wireless channels for maximizing cell throughput under certain fairness constraints. In Temporal Fair (TF) singlecell opportunistic scheduling, the cell throughput is maximized under the constraint that users receive the same share of airtime resources [2]. It was shown in [2] that the optimum $\mathrm{TF}$ scheduler chooses to serve the user which has the largest sum of available transmission rate and another user-dependent term that can be calculated off-line if the channel models are available or alternatively can be obtained using an on-line learning algorithm. A conventional frequency reuse- $M$ system with uniform partitioning of the frequency band and with TF scheduling on a cell-basis is both inter-cell fair (in terms of

Manuscript received September 27, 2015; revised February 6, 2015; accepted February 11, 2015. Date of publication February 24, 2015; date of current version June 18,2015 . The associate editor coordinating the review of this paper and approving it for publication was L. Badia.

S. Shahsavari was with Bilkent University, Ankara 06800, Turkey. He is now with Sharif University, Tehran 11365-9363, Iran (e-mail: shahram.shahsavari. aut.ac@gmail.com).

N. Akar is with Bilkent University, Ankara 06800, Turkey (e-mail: akar@ ee.bilkent.edu.tr).

Color versions of one or more of the figures in this paper are available online at http://ieeexplore.ieee.org.

Digital Object Identifier 10.1109/LWC.2015.2406752 bandwidth) and intra-cell temporal fair. For a survey on singlecell scheduling in LTE networks, see [3].

Frequency reuse-1 (or single channel) networks have gained attention recently in which all cells operate at the same frequency band to maximize spectral efficiency since the peak data rates would be higher per user in such systems [4]. However, inter-cell interference is a major concern especially for cell edge users. To control interference in such networks, dynamic cell coordination-based methods have been proposed which can be implemented in different complexities by centralized, semi-distributed, coordinated-distributed and autonomous-distributed methods surveyed in [5]. Moreover, the single-cell scheduling paradigm is replaced with multi-cell scheduling for which a scheduling decision is to be made while taking into consideration of all cells in the network. For a recent review on multi-cell scheduling, see [6].

In this letter, we consider the downlink of a single-channel network with the transmit powers being fixed for each cell as in most single-cell schedulers. We do not consider sectoring in this letter. We assume a time-slotted system for the sake of convenience but the proposed mechanisms can also be deployed in OFDMA-based systems with modifications. Our goal is to improve the throughput of conventional frequency reuse- $M$ systems while preserving their inter-cell and intra-cell fairness features. For this purpose, we propose a two-level scheduler. At a scheduling instant, each BS employs a cell level TF scheduler to nominate a user and its available transmission rate to the network level. The network level then calculates the potential overall transmission rate for each pattern and runs a network level TF scheduler to decide on which pattern to allow to transmit. This decision is then disseminated to all BSs which then forward data to their nominated users if the pattern they belong to, was chosen for transmission. Both TF schedulers are tuned to provide inter- and intra-cell fairness but designed to maximize the overall network throughput. Low processing requirement of the proposed approach and the limited amount of information exchange among the BSs and the network level scheduler, are the apparent advantages in comparison with other centralized schemes that have higher implementation complexities [5]. We also provide an extension of this scheduler by introducing a virtual user in each cell and additionally a virtual pattern at the network level for network throughput enhancement by relaxing temporal fairness with air-time share guarantees. We present the two-level scheduler in Section II and validate its effectiveness in Section III. Finally, we conclude.

\section{Two-Level Multi-Cell Scheduling Algorithm}

We envision a time-slotted frequency reuse-1 network with bandwidth $B W$ serving $K$ cells where the time slots of a certain duration are indexed by $1 \leq \tau<\infty$. We assume $M$ transmission 
(a)

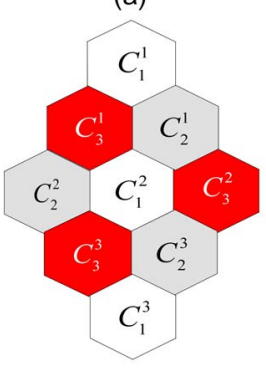

(b)

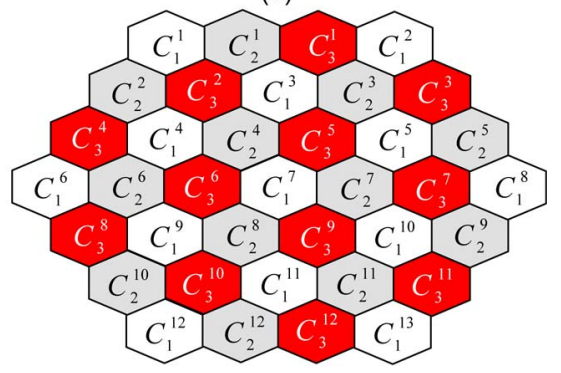

Fig. 1. A frequency reuse-1 network with $M=3$ patterns: (a) 9-cell scenario and (b) 37-cell scenario.

patterns a given cell may belong to. We assume that all cells in a transmission pattern can transmit simultaneously with a mutual interference that is tolerable. The $M$ transmission patterns may be the same as the patterns in conventional frequency reuse$M$ systems which is the approach we pursue in this paper in which case the patterns are mutually disjoint. Use of other transmission patterns of other frequency reuse systems such as FFR (Fractional Frequency Reuse) or PFR (Partial Frequency Reuse) described in [5] is left for future study. Let $K_{m}$ denote the number of cells in pattern $m=1,2, \ldots, M$. Moreover, let $C_{m}^{i}, i=1,2, \ldots, K_{m}$ denote the cell $i$ for pattern $m$. Let $N$ denote the total number of users in the network and let $N_{m}^{i}$ denote the number of users in cell $C_{m}^{i}$. Clearly, $K=$ $\sum_{m} K_{m}$ and $N=\sum_{m} \sum_{i} N_{m}^{i}$. We assume that each user is already associated with a cell. Let $U_{m}^{i, j}$ denote the user $j, j=$ $1,2, \ldots, N_{m}^{i}$ associated with cell $i$ of pattern $m$. We assume all users $U_{m}^{i, j}$ are active and they always have data to send. Fig. 1 illustrates two frequency reuse- 1 network scenarios each with $M=3$ patterns, one with 9 cells $\left(K_{m}=3, m=1,2,3\right)$ and the other with 37 cells $\left(K_{1}=13, K_{m}=12, m=2,3\right)$. Note that these patterns are the same as those of a conventional frequency reuse- 3 network.

At a given time slot $\tau$, the two-level scheduler will first choose opportunistically a pattern out of the $M$ available and then all cells in that pattern will choose a user opportunistically for downlink transmission. For fairness constraints, we let $a_{m}^{i, j}(t)\left(A_{m}(t)\right)$ denote the cumulative air-time share of user $U_{m}^{i, j}$ (pattern $m$ ) up to time $t$, i.e., $a_{m}^{i, j}(t)\left(A_{m}(t)\right)=$

$$
\frac{1}{t} \sum_{\tau=1}^{t} I\left\{\mathrm{User} U_{m}^{i, j}(\text { Pattern } m) \text { is selected at slot } \tau\right\},
$$

where $I\{\cdot\}$ denotes the conventional indicator function which is either one or zero depending on whether the argument is true or not, respectively. We define the long-term air-time shares of user $U_{m}^{i, j}$ and pattern $m$ by $a_{m}^{i, j}=\lim _{t \rightarrow \infty} a_{m}^{i, j}(t)$, and $A_{m}=\lim _{t \rightarrow \infty} A_{m}(t)$, respectively. We introduce positive scheduling weights $W_{m}$ and $w_{m}^{i, j}$, for pattern $m$ and for user $U_{m}^{i, j}$, respectively, satisfying $\sum_{m} W_{m}=1$ and $\sum_{j} w_{m}^{i, j}=1$ for any given cell $C_{m}^{i}$. When $A_{m}=W_{m}$, for all $m$, then the system is inter-cell weighted temporal fair with respect to the weights $\left\{W_{m}\right\}$ since each cell belongs to one pattern only. Similarly, when $a_{m}^{i, j}=A_{m} w_{m}^{i, j}$ for a given cell $C_{m}^{i}$, then we have intracell weighted temporal fairness in cell $C_{m}^{i}$ with respect to the weights $\left\{w_{m}^{i, j}\right\}$. Weighted inter- and intra-cell temporal fairness reduce to ordinary temporal fairness with the choice of the weights $W_{m}=W_{m^{\prime}}, \forall m, m^{\prime}$ and $w_{m}^{i, j}=w_{m}^{i, j^{\prime}}, \forall j, j^{\prime}$ for each cell $C_{m}^{i}$, respectively. For fairness purposes, we introduce a counter $b_{m}^{i, j}$ for each user $U_{m}^{i, j}$ and another counter $B_{m}$ for each pattern $m$. We set these counter values to zero at the beginning of network operation. We also define the instantaneous Spectral Efficiency $(\mathrm{SE}) r_{m}^{i, j}(\tau)$ in units of bits/s/Hz for user $U_{m}^{i, j}$ at time slot $\tau$. In particular, in our numerical experiments, we use the Shannon formula

$$
r_{m}^{i, j}(\tau)=\log _{2}\left(1+S N R_{m}^{i, j}(\tau)\right)
$$

where $S N R_{m}^{i, j}(\tau)$ denotes the signal to noise ratio of user $U_{m}^{i, j}$ at slot $\tau$ [7] but also other relationships of SE to the SNR than (2) can also be used. Next, we describe the two-level multi-cell scheduler algorithm we propose at a given time slot $\tau$. At the cell level, the BS of cell $C_{m}^{i}$ selects a user $j_{i, m}^{*}$ based on the instantaneous SEs and the user counter values as follows:

$$
j_{i, m}^{*}:=\underset{j \in\left\{1,2, \ldots, N_{m}^{i}\right\}}{\arg \max }\left(r_{m}^{i, j}(\tau)+\alpha b_{m}^{i, j}\right),
$$

where $\alpha>0$ is an algorithm parameter that we will study in the numerical examples which will be shown to affect the convergence time and the overall network throughput. Each BS of cell $C_{m}^{i}$ then nominates the user $j_{i, m}^{*}$ and the instantaneous SE of the cell denoted by $R_{m}^{i}(\tau):=r_{m}^{i, j_{i, m}^{*}}(\tau)$ if the nominated user were to be served. In the second step, the BSs of cells $C_{m}^{i}$ disseminate the values $R_{m}^{i}(\tau)$ to the network-level. In the third step, the network-wide SE of pattern $m$ denoted by $R_{m}(\tau)$ is obtained as follows:

$$
R_{m}(\tau):=\sum_{i=1}^{K_{m}} R_{m}^{i}(\tau), m=1,2, \ldots, M .
$$

In the fourth step, the network level selects the pattern $m^{*}$ on the basis of the following identity:

$$
m^{*}:=\underset{m \in\{1,2, \ldots, M\}}{\arg \max }\left(R_{m}(\tau)+\beta B_{m}\right),
$$

where $\beta>0$ is again an algorithm parameter. Once $m^{*}$ is determined, the network level counters are updated in the fifth step as follows:

$$
B_{m}:=B_{m}+W_{m}-I\left\{m=m^{*}\right\}, m=1,2, \ldots, M .
$$

The network level then sends a command to all cells with the information on which pattern was selected in the current slot. In the final step of the algorithm, the cells $C_{m^{*}}^{i}, i=1,2, \ldots, K_{m^{*}}$ transmit to their nominated users $j_{i, m^{*}}^{*}$, respectively, with an SE of $r_{m^{*}}^{i, j_{i, m^{*}}^{*}}(\tau)$ and the user counters of cells $C_{m^{*}}^{i}, i=$ $1, \ldots, K_{m^{*}}$, are updated as follows:

$$
b_{m^{*}}^{i, j}:=b_{m^{*}}^{i, j}+w_{m^{*}}^{i, j}-I\left\{j=j_{i, m^{*}}^{*}\right\}, j=1,2, \ldots, N_{m^{*}}^{i}
$$

The counters of users in cells in patterns other than the selected one are not updated and those cells are switched off in the current slot. The network level scheduler has $\mathcal{O}(K)$ 
computational complexity and $\mathcal{O}(M)$ storage requirements and presents a scalable solution when compared to existing methods whose complexity depend on the overall number of users $N$ in the network. Due to low communications overhead between the BSs and network level scheduler, the proposed method is practical and can be implemented using a high-speed backhaul. The proposed scheme can be viewed as a two-level credit-based procedure where chosen (un-chosen) patterns loose (gain) credits and served (un-served) users of the chosen pattern again loose (gain) credits and the algorithm parameters $\alpha$ and $\beta$ are the weights of the credit component at the cell and networks levels, respectively. The overall long-term average network throughput is given by $T=\lim _{t \rightarrow \infty} \frac{B W}{t} \sum_{\tau=1}^{t} R_{m^{*}}(\tau)$. The proposed algorithm provides weighted inter- and intra-cell temporal fairness due to the way counters are updated. Scheduling decisions are always made to ensure that counter values stay bounded in absolute value which can be shown to guarantee the long-term inter- and intra-cell weighted temporal fairness constraints. The optimality of the proposed multi-cell scheduler stems from the structure of the two TF schedulers, the networklevel TF scheduler (5) and the cell-level TF scheduler (3), which are the same as the single-cell optimum TF scheduler described in [2] except that we use fixed coefficients $\beta$ and $\alpha$ in the two TF schedulers instead of those that decay in time. The purpose of this choice is to satisfy fairness constraints not only in the long term but also in shorter time scales. Numerical examples will be presented to validate these choices.

Finally, we present an extension of the proposed algorithm with the intention of increasing overall network throughput while relaxing weighted inter- and intra-cell temporal fairness with air-time share guarantees. For this purpose, we introduce a virtual user denoted by $v_{m}^{i}$ for each cell $C_{m}^{i}$. The SE of the virtual user $v_{m}^{i}$ at slot $\tau$ is set to the maximum instantaneous SE of all users in cell $C_{m}^{i}$. A scheduling weight $w_{m}^{i}$ is assigned to the virtual user satisfying $\sum_{j=1}^{N_{m}^{i}} w_{m}^{i, j}+w_{m}^{i}=1$ and a counter denoted by $b_{m}^{i}$ is dedicated to the virtual user $v_{m}^{i}$. Similarly, a virtual pattern $V$ is introduced whose $\mathrm{SE}$ at slot $\tau$ is set to the maximum SE of all patterns. A scheduling weight $W_{v}$ is assigned to the virtual pattern satisfying $\sum_{m=1}^{M} W_{m}+W_{v}=1$. Finally, a counter denoted by $B_{v}$ is dedicated to virtual pattern $V$. The previously proposed two-level scheduler is run as is, with the additional virtual pattern and per-cell virtual users. At a scheduling instant $\tau$, when the network-level scheduler is to choose the virtual pattern, the pattern that is actually allowed to transmit would be the one that has the largest per-pattern SE. When the cell-level scheduler is to choose a virtual user, then the user with the maximum SE in that cell would be chosen for transmission. It is clear that the following inequalities $A_{m} \geq$ $W_{m}$ and $a_{m}^{i, j} \geq w_{m}^{i, j} W_{m}$ hold as opposed to equalities provided by the original algorithm in the absence of virtual users and virtual pattern. When a virtual pattern or virtual user is selected, only their dedicated counters are updated and not those of the actual pattern and users that are chosen for transmission. Consequently, with this new enhancement, weighted temporal fairness constraints are replaced with weighted air-time share guarantees with suitable choices of weights in the network. We call this algorithm TLSAG (Two Level Scheduler with Air-time Guarantees) as opposed to the original algorithm TLSTF (Two
Level Scheduler with Temporal Fairness) which refers to the case $W_{v}=0, w_{m}^{i}=0$, for all cells $C_{m}^{i}$.

\section{NUMERICAL EXAMPLES}

In all the numerical examples, we use the 9-cell and 37-cell frequency reuse-1 networks depicted in Fig. 1 where the radius of each cell is $1 \mathrm{~km}$. The system frequency is assumed to be $2.5 \mathrm{GHz}$ and $B W$ is set to $60 \mathrm{MHz}$. For the benchmark frequency reuse- 3 system using $20 \mathrm{MHz}$ subbands, noise power and all BS transmit powers are set to $-100 \mathrm{dBm}$ and $45 \mathrm{dBm}$, respectively. Rayleigh fading and log-distance path-loss models are adopted for the channel distribution of each user [1]. The path-loss exponent and the Rayleigh parameters are set to 3.5 and 2.5, respectively. For the sake of convenience, we set $w_{m}^{i, j}=$ constant, for all $j$ in any given cell $C_{m}^{i}$ and $W_{m}=$ constant for all patterns $m$ in the numerical examples, i.e., we seek ordinary temporal fairness. For a given cell $C_{m}^{i}$, let $J_{m}^{i}(t)$ denote the Jain's fairness index (see [8] for the definition) for the values $a_{m}^{i, j}(t)$ and let us define the intra-cell fairness index $J^{*}(t)=\min _{m, i} J_{m}^{i}(t)$. Also let the inter-cell fairness index $J(t)$ be defined by Jain's fairness index for the individual perpattern air-time shares $A_{m}(t)$. Proximity of $J^{*}(t)$ and $J(t)$ to unity are representative of intra- and inter-cell fairness, respectively, up to time $t$.

In the first example, we assume $N_{m}^{i}=10$ uniformly located users in each cell. We run the TLSTF algorithm for a duration of $5 \times 10^{6}$ slots with various choices of $\alpha$ and $\beta$ and obtain the values $\Gamma_{J}$ and $\Gamma_{J^{*}}$, which are defined as the minimum value of $t$ such that $J(H t)>1-\varepsilon$ and $J^{*}(H t)>1-\varepsilon$, respectively, for a small tolerance parameter $\varepsilon>0$ which is set to 0.005 , and for a sampling parameter $H$ set to 100 . Larger values of $\Gamma_{J}$ and $\Gamma_{J^{*}}$ are indicative of longer convergence times and therefore adverse impact on short-term inter- and intra-cell fairness, respectively. The steady-state throughput $T$, and two fairness metrics $\Gamma_{J}$ and $\Gamma_{J^{*}}$ are tabulated in Table I for various values of $\alpha$ and $\beta$ and for two network scenarios. We observe that with relatively low choices of the algorithm parameters $\alpha$ and $\beta$, it takes longer for the system to be inter- and intra-cell temporal fair. Intra-cell short-term fairness appears to be more problematic. On the other hand, when these parameters are increased, the throughput $T$ of the system is slightly reduced. Moreover, the choice of $\alpha$ has a far larger impact on system performance than $\beta$. As a trade-off between short-term fairness and total network throughput, we fix $\alpha=\beta=0.01$ in the remaining numerical examples.

In the second numerical example, we vary $N$ and for each value of $N$, we create 300 instances in each of which $N$ users are randomly spatially distributed in the 9-cell and 37cell network scenarios. For each instance, we define the percentage gain $G$ as $\frac{T_{T L S T F R 3}-T_{F R 3}}{T_{F R}} 100 \%$ where $T_{T L S T F}$ and $T_{F R 3}$ denote the total network throughput $T$ obtained by using TLSTF and the frequency reuse-3 system, respectively, under the same average transmit power, with each cell deploying the cell level scheduler of TLSTF independently of other cells in the latter system. We plot the empirical Cumulative Distribution Function (CDF) of the percentage gain $G$ in Fig. 2 for five different scenarios with the minimum (maximum) observed 
TABLE I

The Throughput $T$, And Two Fairness Metrics $\Gamma_{J}$ And $\Gamma_{J}{ }^{*}$, FOR VARIOUS VALUES OF $\alpha$ AND $\beta$ FOR THE TWO NETWORK SCENARIOS

\begin{tabular}{|c|c|c|c|c|c|}
\hline Scenario & $\alpha$ & $\beta$ & $T$ (Gbps) & $\overline{\Gamma_{J}}$ & $\Gamma_{J} *$ \\
\hline \multirow{16}{*}{ 9-cell } & \multirow{4}{*}{0.001} & 0.001 & 0.448 & 1331 & 30796 \\
\hline & & 0.005 & 0.447 & 606 & 29564 \\
\hline & & 0.01 & 0.447 & 323 & 29553 \\
\hline & & 0.05 & 0.446 & 70 & 29543 \\
\hline & \multirow{4}{*}{0.005} & 0.001 & 0.425 & 368 & 6857 \\
\hline & & 0.005 & 0.424 & 270 & 6140 \\
\hline & & 0.01 & 0.424 & 175 & 5919 \\
\hline & & 0.05 & 0.424 & 65 & 5911 \\
\hline & \multirow{4}{*}{0.01} & 0.001 & 0.421 & 208 & 3451 \\
\hline & & 0.005 & 0.421 & 155 & 3247 \\
\hline & & 0.01 & 0.421 & 134 & 3065 \\
\hline & & 0.05 & 0.421 & 65 & 2956 \\
\hline & \multirow{4}{*}{0.05} & 0.001 & 0.418 & 86 & 699 \\
\hline & & 0.005 & 0.417 & 41 & 693 \\
\hline & & 0.01 & 0.417 & 37 & 689 \\
\hline & & 0.05 & 0.417 & 27 & 617 \\
\hline \multirow{16}{*}{ 37-cell } & \multirow{4}{*}{0.001} & 0.001 & 1.215 & 1521 & 39212 \\
\hline & & 0.005 & 1.212 & 1117 & 36781 \\
\hline & & 0.01 & 1.211 & 630 & 36033 \\
\hline & & 0.05 & 1.210 & 164 & 35187 \\
\hline & \multirow{4}{*}{0.005} & 0.001 & 1.158 & 415 & 8246 \\
\hline & & 0.005 & 1.157 & 303 & 7871 \\
\hline & & 0.01 & 1.157 & 270 & 7622 \\
\hline & & 0.05 & 1.156 & 125 & 7224 \\
\hline & \multirow{4}{*}{0.01} & 0.001 & 1.149 & 264 & 4203 \\
\hline & & 0.005 & 1.149 & 171 & 4048 \\
\hline & & 0.01 & 1.149 & 153 & 3935 \\
\hline & & 0.05 & 1.149 & 113 & 3719 \\
\hline & \multirow{4}{*}{0.05} & 0.001 & 1.138 & 252 & 843 \\
\hline & & 0.005 & 1.138 & 55 & 835 \\
\hline & & 0.01 & 1.138 & 44 & 818 \\
\hline & & 0.05 & 1.138 & 31 & 801 \\
\hline
\end{tabular}

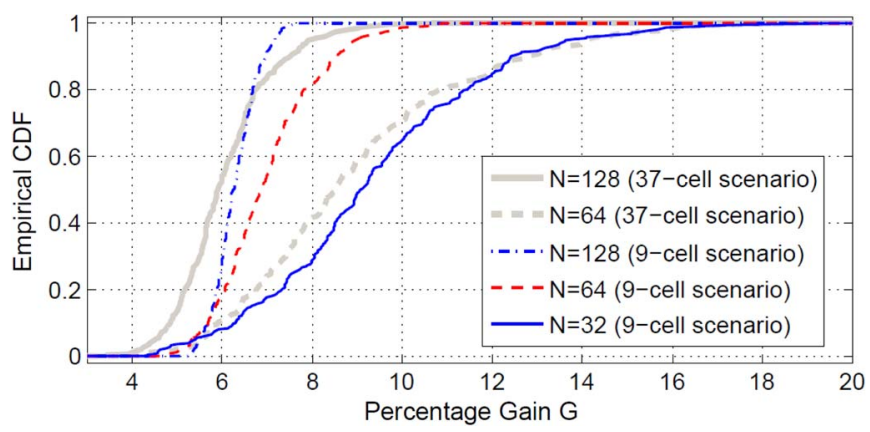

Fig. 2. The empirical CDF of the percentage gain $G$ for various scenarios.

gain being $3.45 \%$ (19.48\%). We observe that the gain $G$ appears to increase with decreasing average number of users per cell in which case the multi-user diversity gain is limited with single-cell scheduling. However, in such scenarios, networkwide multi-user diversity due to multi-cell scheduling helps improve the total network throughput.

In the final example, we assess the throughput performance of TLSAG. $N_{m}^{i}=10$ users are located uniformly in each cell in this example. We set the virtual user weight $w_{m}^{i}=w$ and $w_{m}^{i, j}=(1-w) / 10$ for all cells $C_{m}^{i}$ and we vary the weight of the virtual user $w$ and that of the virtual pattern $W_{v}$ in the interval $[0,0.3]$ with a step size of 0.02 for which $W_{m}=(1-$ $\left.W_{v}\right) / 3, m=1,2,3$. Note that TLSAG reduces to TLSTF when $w=W_{v}=0$. We run the TLSAG algorithm for $10^{6}$ time slots and plot the percentage improvement in network throughput obtained with TLSAG in comparison with TLSTF, as a function (a)

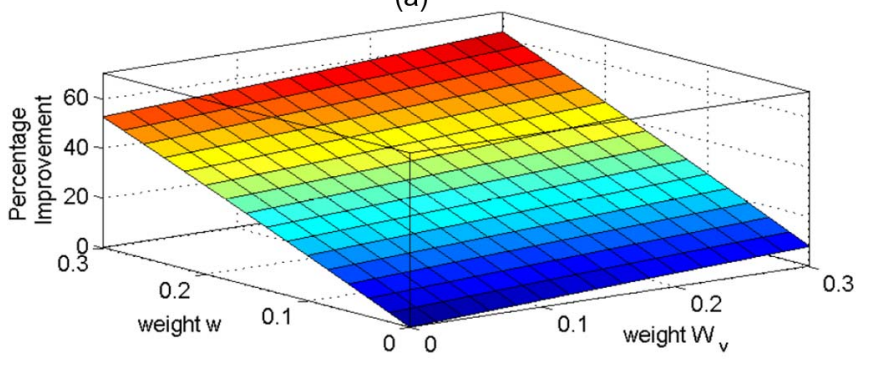

(b)

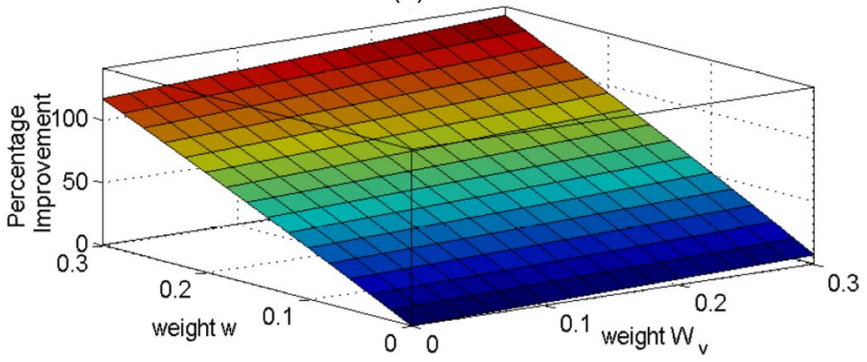

Fig. 3. Percentage improvement in $T$ attained with TLSAG compared with TLSTF as a function of $w$ and $W_{v}$ : (a) 9-cell and (b) 37-cell scenarios.

of the weights $w$ and $W_{v}$ in Fig. 3. We show that the gain in network throughput linearly increases with these two weights with gains of $62.06 \%$ and $132.96 \%$ when $w=W_{v}=0.3$ for the 9-cell and 37-cell scenarios, respectively. Note that for this particular scenario, TLSAG ensures that $a_{m}^{i, j} \geq \frac{(1-0.3)^{2}}{30}$, as opposed to TLSTF which yields $a_{m}^{i, j}=\frac{1}{30}$, for all users $U_{m}^{i, j}$.

\section{CONCLUSION}

A novel scheduler TLSTF is proposed for a frequency reuse1 multi-cell wireless network providing inter- and intra-cell (weighted) temporal fairness while maximizing overall network throughput. We also propose an extension, called TLSAG, which provides long term temporal share guarantees to users. Notable gains are demonstrated with both algorithms.

\section{REFERENCES}

[1] T. Rappaport, Wireless Communications: Principles and Practice, 2nd ed. Upper Saddle River, NJ, USA: Prentice-Hall, 2001.

[2] X. Liu, E. K. P. Chong, and N. Shroff, "Opportunistic transmission scheduling with resource-sharing constraints in wireless networks," IEEE J. Sel. Areas Commun., vol. 19, no. 10, pp. 2053-2064, Oct. 2001.

[3] F. Capozzi, G. Piro, L. Grieco, G. Boggia, and P. Camarda, "Downlink packet scheduling in LTE cellular networks: Key design issues and a survey," IEEE Commun. Surveys Tuts., vol. 15, no. 2, pp. 678-700, Second Quart. 2013.

[4] S.-E. Elayoubi, O. Ben Haddada, and B. Fourestie, "Performance evaluation of frequency planning schemes in OFDMA-based networks," IEEE Trans. Wireless Commun., vol. 7, no. 5, pp. 1623-1633, May 2008.

[5] A. Hamza, S. Khalifa, H. Hamza, and K. Elsayed, "A survey on inter-cell interference coordination techniques in OFDMA-based cellular networks," IEEE Commun. Surveys Tuts., vol. 15, no. 4, pp. 1642-1670, Fourth Quart. 2013.

[6] E. Pateromichelakis, M. Shariat, A. ul Quddus, and R. Tafazolli, "On the evolution of multi-cell scheduling in 3GPP LTE/LTE-A," IEEE Commun. Surveys Tuts., vol. 15, no. 2, pp. 701-717, Second Quart. 2013.

[7] D. Tse and P. Viswanath, Fundamentals of Wireless Communication. New York, NY, USA: Cambridge Univ. Press, 2005.

[8] R. Jain, The Art of Computer Systems Performance Analysis-Techniques for Experimental Design, Measurement, Simulation, Modeling. Hoboken, NJ, USA: Wiley, 1991, ser. Wiley Professional Computing. 